\title{
Eye movements in vocabulary research
}

\author{
Ana Pellicer-Sánchez and Anna Siyanova-Chanturia \\ UCL Institute of Education, UK |Victoria University of Wellington, \\ New Zealand
}

The field of vocabulary research is witnessing a growing interest in the use of eye-tracking to investigate topics that have traditionally been examined using offline measures, providing new insights into the processing and learning of vocabulary. During an eye-tracking experiment, participants' eye movements are recorded while they attend to written or auditory input, resulting in a rich record of online processing behaviour. Because of its many benefits, eye-tracking is becoming a major research technique in vocabulary research. However, before this emerging trend of eye-tracking based vocabulary research continues to proliferate, it is important to step back and reflect on what current studies have shown about the processing and learning of vocabulary, and the ways in which we can use the technique in future research. To this aim, the present paper provides a comprehensive overview of current eye-tracking research findings, both in terms of the processing and learning of single words and formulaic sequences. Current research gaps and potential avenues for future research are also discussed.

Keywords: eye movements, vocabulary, formulaic language, lexical processing, vocabulary learning.

\section{Introduction}

Eye-tracking has been extensively used in psycholinguistic research to explore the processing of written and auditory stimuli. Thus, a wealth of research on the processing of different types of lexical items is currently available. This research has provided a rich (although not yet complete) picture of how different types of lexical items are processed by first (L1) and second language (L2) speakers, as well as of the effects of different factors on this process.

Researchers exploring vocabulary learning have traditionally used offline measures (such as vocabulary tests and think aloud protocols). However, recent interdisciplinary research has started to use eye-tracking to investigate different 
aspects of the vocabulary learning process, giving rise to a new trend in vocabulary research. Although still in its incipient stage, important insights into the relationship between the processing and learning of lexical items have already been gained. We are witnessing an increase in interdisciplinary studies using eye-tracking to explore not only the processing and learning of vocabulary, but also the relationship between the two. We believe that, in order to advance the field and to consolidate this emerging line of research, it is important to provide vocabulary researchers with an overview of the current findings and possible directions for future research.

In this review, we cover research in relation to both single words and formulaic sequences. It is important to note that we use the term 'formulaic language' as the umbrella term and 'formulaic sequences' to refer to individual instances of formulaic language. Where appropriate, specific types of formulaic language are referred to by their own names such as collocations, idioms, binomials, and so forth. The review is structured as follows. First, we briefly introduce the eye-movement methodology. We then review in some detail the eye-tracking studies on the processing of single words and formulaic sequences, followed by a review of what eye-tracking studies have revealed about the learning of single words and formulaic sequences. Finally, we discuss some directions for future research on vocabulary employing eye movements.

\section{The eye-tracking methodology}

Eye-tracking allows researchers to examine participants' eye movements while processing different types of verbal (written and auditory) and visual stimuli. It provides a record of what exactly participants are looking at and for how long. It measures the periods of relative stability of the eyes where information is extracted (fixations) and the rapid movements between fixations (saccades). In the processing of written input, regressive eye movements (movements to an earlier text) are also recorded (see Figure 1). Together, these data provide a rich, real-time record of online processing behaviour. Unlike other tasks that measure processing time, eye-tracking does not require a secondary task, such as a button press in a lexical decision task or an oral response in a naming task. For these reasons, researchers have referred to eye-tracking studies as a better representation of natural reading behaviour (Conklin \& Pellicer-Sánchez, 2016). In addition, unlike other measures of reading time, eye-tracking allows researchers to present critical items (single words or phrases) in sentence or story contexts, allowing for a more natural reading experience (compared to a word-by-word self-paced reading). 


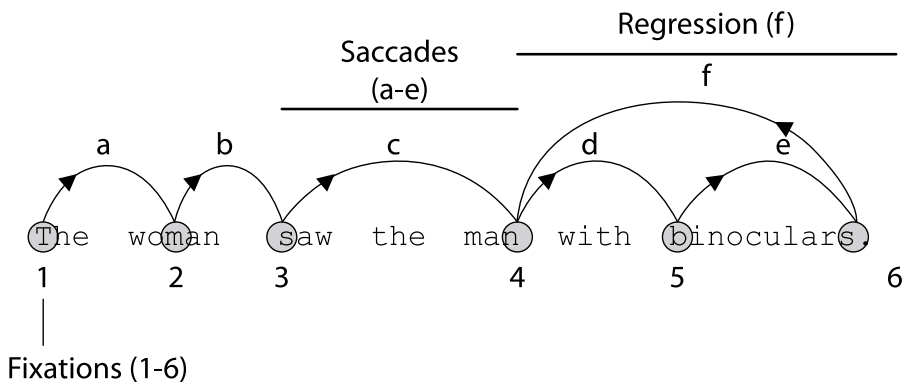

Figure 1. Hypothetical patterns of eye movements during reading depicting fixations (grey circles), saccades (forward curves) and a regressive movement (backword curve)

Eye-movement data are normally reported as fixations within a particular area of interest (AOI) and the duration of these fixations. The underlying assumption is that the longer the fixations and the higher the number of fixations made within a given AOI, the higher the cognitive effort needed to process the information. A range of measures can be used to analyse eye movements. A distinction is often made between temporal measures (e.g. fixation duration) which indicate processing load, and spatial measures (e.g. fixation position) which are believed to indicate direction of processing (Radach \& Kennedy, 2004). A further distinction within temporal measures is made between early and late measures. Early measures reflect the initial stages of processing and are an indication of more automatic processing, whereas late measures indicate more controlled and strategic processing. Common early measures include: first fixation duration (the duration of the first fixation made within an AOI), and first pass reading time (the duration of all fixations made within an AOI before exiting the area either to the left or to the right). Commonly reported late measures are: fixation count (number of fixations made within an AOI), second pass reading time (the sum of the duration of all fixations made within an AOI after it has been exited for the first time), and total fixation duration or total reading time (the sum of the duration of all fixations made within an AOI). In addition, eye movements are often reported in terms of the likelihood of skipping of a particular AOI, which is another early eye-movement measure (see Conklin \& Pellicer-Sánchez, 2016, for a review of other measures).

In vocabulary studies, the early measures reflect the earlier stages of lexical access and lexical processing, such as word recognition, whereas late measures are said to reflect higher-level processes of lexical integration. Shorter fixations, fewer fixations, and a higher skipping rate in target lexical items are interpreted as an indication of less cognitive effort involved and less processing difficulty. It is noteworthy that a variety of measures is typically analysed and reported in 
order to obtain a full picture of early and later processes; using only one measure can provide only a very incomplete account of language processing (Rayner, 2009). The studies reviewed below show, among other things, differential effects depending on the measure explored. For example, in the study by Vilkaite (2016) on the processing of non-adjacent collocations (described below), collocational status was a significant predictor of total reading time and fixation count (late measures), but did not significantly predict first fixation duration and first pass reading time (early measures).

\section{Processing of single words}

In this section, we turn to some of the major factors known to affect lexical - single word - processing in L1 and L2. ${ }^{1}$

As noted above, one of the advantages of the eye-movement method is the ability to determine what was fixated, for how long, how many times, and in what order. However, this method allows us to study not only what was fixated during reading, but also what was skipped. A wealth of eye-tracking studies in the L1 context have shown that higher frequency and shorter words are more likely to be skipped than lower frequency and longer words. Function words, which are normally frequent and short, are fixated only $35 \%$ of the time (Carpenter \& Just, 1983). It is estimated that around 15\% of content words are skipped during natural reading (Carpenter \& Just, 1983). The word's frequency, length and predictability are some of the major factors affecting how the word is read (for a review, see Rayner, 2009). For example, Inhoff and Rayner (1986) and Rayner and Duffy (1986) showed that higher frequency words (music) were recognised faster than lower frequency words of the same length (waltz). Further, more predictable words (given the preceding context), are more likely to receive fewer and shorter fixations, or skipped altogether, compared to unexpected words (Balota et al., 1985; Kliegl et al., 2004; Rayner \& Well, 1996). For example, the word cake in the sentence Since the wedding was today, the baker rushed the wedding ... to the reception was read faster than the word pies (Balota et al., 1985).

It is important to note that if a word has not been fixated, it does not imply that it has not been processed. Higher frequency, shorter, and more predictable words are more likely to be processed in the parafovea (the area that precedes the word in question) - and, thus, skipped - when compared to lower frequency, longer, and unexpected words (Inhoff \& Rayner, 1986; Rayner, 2009). Given

1. Note on terminology: we use L1 and L2 speakers interchangeably with monolinguals and bilinguals. 
that the size of the perceptual span (i.e., the amount of information that can be extracted during one fixation) in alphabetical languages is $14-15$ characters to the right of a given fixation (McConkie \& Rayner, 1975; Rayner \& Bertera, 1979), it is possible for one or more words to be processed parafoveally and receive no fixations.

Although the effects of frequency have for the most part been investigated in the context of L1, some studies have also looked at word frequency effects in bilinguals. In the L1 context, high frequency words (e.g., table) are recognised faster and more accurately than low frequency words (e.g., patio). In the context of bilingualism, greater L2 exposure should strengthen L2 lexical representation and reduce L2 word frequency effects, while reduced L1 exposure should weaken L1 lexical representation and increase L1 word frequency effects (see Whitford, Pivneva \& Titone, 2016). This is because, as explained by Whitford et al. (2016), increased exposure to a low frequency word reduces the time it takes to recognise it, while greater exposure to a high frequency word only marginally affects its lexical access time. The studies employing response-based tasks (e.g., lexical decision task) have shown that L2 word frequency effects in bilinguals are larger than L1 word frequency effects in monolinguals and L1 word frequency effects in bilinguals (for a review, see Whitford, et al., 2016). The eye-tracking studies have added to our understanding of word frequency effects in sentential, and hence more naturalistic, contexts across bilinguals' $\mathrm{L}_{1}$ and L2, as well as pointed to an important role of L2 exposure. For example, Whitford and Titone (2012) found larger L2 than L1 word frequency effects in a group of L1-dominant bilinguals. This was the case for both early (e.g., first pass reading time) and late (e.g., total reading time) eye-tracking measures, reflecting initial lexical access and post-lexical access, respectively. Interestingly, this effect was modulated by the differences in bilinguals' L2 exposure. Bilinguals with higher exposure to L2 showed smaller L2 word frequency effects than bilinguals with lower L2 exposure, but larger L1 word frequency effects (Whitford \& Titone, 2012).

3.1 Cross-language activation: Cognates, interlingual homographs and orthographic neighbourhood

While the factors reviewed above have been examined in the context of L1 and, to a lesser extent, L2 processing, other factors have received attention specifically in the context of L2 word recognition. A central question in the study of L2 lexical processing has been the issue of cross-language activation, and whether word recognition involves activation of lexical candidates from the non-target language (Desmet \& Duyck, 2007; Dirix et al., 2017; Whitford et al., 2016). 
As noted by Desmet and Duyck (2007), according to one account of bilingual language processing, bilinguals have two separate, independent lexicons, one that contains the words in L1 and one that contains the words in L2. In this account, lexical processing is language selective. The other possibility is that lexical representations from $\mathrm{L}_{1}$ are activated during the processing of words from $\mathrm{L} 2$, and vice versa (Desmet $\&$ Duyck, 2007). In this account, lexical processing is language nonselective. A wealth of behavioural studies, employing response-based tasks, have provided evidence in support of the latter, showing that the languages of a bilingual speaker necessarily influence each other during processing. Critical evidence has come from the studies on cognates, ${ }^{2}$ showing that cognates enjoy a processing advantage compared to non-cognates (Lemhöfer, Dijkstra, \& Michel, 2004), and interlingual homographs, ${ }^{3}$ showing that a homograph's processing in one language leads to its activation in the other language (von Studnitz \& Green, 2002). The eyetracking methodology, however, has allowed researchers to examine cross-language activation during more naturalistic reading, in bilingual's L2 and to a lesser extent in their L1, with target words appearing in sentence contexts, rather than in isolation. As argued by Roberts and Siyanova-Chanturia (2013), "a more finegrained picture of bilingual lexical processing can be obtained using eye-tracking as compared to other methods and ... the results can better inform theories about the nature of the bilingual mental lexicon" (p.224).

A number of eye-movement studies have examined cross-language activation by means of cognates and interlingual homographs (Whitford et al., 2016). A dominant finding has been that bilinguals read cognates in sentence contexts faster than matched language-unique controls (e.g., ring is a piece of jewelry in English and Dutch; coat is an English-only word, hence ring should be read faster than coat [example from Duyck et al., 2007]). The use of eye movements has allowed researchers to observe this processing advantage, known as cognate facilitation effect, from the earliest stages of word processing (Duyck, Van Assche, Drieghe, \& Hartsuiker, 2007; Libben \& Titone, 2009; Van Assche, Duyck, Hartsuiker, \& Diependaele, 2009). The studies on interlingual homographs in sentential contexts suggest faster processing for language-specific controls, the effect that is known in the bilingual literature as interlingual homograph interference (Libben

2. Cognates are words in two (or more) languages that share the same origin. They may or may not share the same (exact) meaning. For example, consider education in English and educación in Spanish, which have a similar meaning; and embarrassed in English and embarazada in Spanish (English translation "pregnant"), which do not share the same meaning.

3. Interlingual homographs are words that exist in two (or more) languages that are identical in form but different in meaning. For example, consider pain in English and in French (meaning "bread"). 
\& Titone, 2009; Titone, et al., 2011). Again, this effect has been observed from the earliest stages of word processing. In addition, the use of context has allowed researchers to bias either the L1 or L2 meaning of target words and examine how contextual constrains (high vs. low) modulate cross-language activation (Libben \& Titone, 2009; Titone, et al., 2011; Van Assche, et al., 2011). For example, Titone et al. (2011) found that high contextual constraint weakened cross-language activation for cognates. It is noteworthy that the effect of contextual constraint has been observed largely during the later stages of reading. Finally, L2 age of acquisition has also been found to impact the degree of non-selective lexical access. Titone et al. (2011) found that the cognate facilitation effect varied as a function of participants' age of acquisition: participants demonstrated greater cognate facilitation when $\mathrm{L} 2$ was acquired earlier in life.

Another line of evidence comes from the studies investigating cross-language orthographic neighbourhood. Orthographic neighbourhood is the total number of words that can be generated by changing one letter in a given word preserving letter positions (Coltheart, Davelaar, Jonasson, \& Besner, 1977). For example, the neighbourhood of mouse is house, louse, rouse, douse, and moose. The prevailing finding in the monolingual literature has been facilitation of neighbourhood size (the number of neighbours) in lexical decision tasks. That is, better performance, in terms of the speed and accuracy of lexical decision, for words with large neighbourhoods (Carreiras et al., 1997; Pollatsek et al., 1999). Eye-tracking has also been fruitfully used to explore the role of neighbourhood size and the frequency of orthographic neighbours (Perea \& Pollatsek, 1998; Pollatsek, Perea, \& Binder, 1999), although the evidence has proven to be mixed. Pollatsek et al. (1999) found longer first pass reading time and total reading time for words with more orthographic neighbours than words with fewer neighbours. This is an interesting finding that appears contrary to the earlier studies employing the lexical decision task (Carreiras et al., 1997; Pollatsek et al., 1999). In addition, Pollatsek et al. (1999) found that if a lower frequency target word had a higher frequency neighbour, the reading of the target was inhibited in later processing stages (also see Perea \& Pollatsek, 1998). This effect is known as the neighbourhood frequency effect and suggests that the higher frequency neighbour may be activated more easily (due to its frequency) and may thus act as a competitor to the lower frequency target (Grainger, O'Regan, Jacobs, \& Segui, 1989).

In a bilingual context, van Heuven, Dijkstra, and Granger (1998) found that word recognition depended on the neighbourhood size not only in a bilingual's L2 (when reading in L2) but also in their L1. In a series of experiments with DutchEnglish bilinguals, these authors showed that lexical orthographic representations from one's L1 remain active during word recognition in one's L2. More recently, Dirix et al. (2017) replicated the findings of van Heuven et al. (1998) using a gen- 
eralised lexical decision task (Experiment 1), and, importantly, extended them using a large-scale eye-movement corpus of late Dutch-English bilinguals when reading L1 and L2 nouns (Experiment 2). Using eye movements, these authors observed cross-language effects of neighbours during bilingual reading in L2 and to a lesser extent in L1 reading, offering strong support to the non-selective lexical activation view. Critically, the eye-tracking data suggested effects of crosslingual neighbourhood in both early and late measures. Interestingly, unlike the results of Experiment 1 where inhibition was observed, the cross-lingual effects found in Experiment 2 were facilitatory, leading to shorter, rather than longer, fixation duration for nouns with more neighbours. It appears that during a lexical decision task, the cross-lingual activation slows performance, while more naturalistic language reading in fact benefits from this cross-lingual activation. As the authors point out, the lexical decision task involves a strong decision component that can result in participants adopting a range of strategies, potentially affecting the nature of lexical access. On the contrary, eye movements allow for more naturalistic reading with minimal instruction on behalf of the researcher, and "without interference from the additional decision components or response mechanisms that are inherent to lexical decision tasks" (Cop et al., 2017, p. 603). Irrespective of the methodology, both experiments pointed to the conclusion that written words activate not only orthographically similar words in the target, but also words in the non-target language (Dirix et al., 2017).

In sum, the studies reviewed in this section have shown that a range of factors have been found to affect monolingual and bilingual lexical processing, such as, frequency, length, predictability, orthographic neighbourhood, as well as cognate and interlingual homograph status. While behavioural methods have been instrumental in obtaining a rich picture of word processing in isolation (using responsebased tasks), the eye-tracking methodology has been key to investigating lexical access in a L1 and L2 and cross-language activation in L2 during more naturalistic, contextual reading with a particular focus on teasing apart early versus late stages of language processing.

\section{Processing of formulaic language}

While there is a long-standing tradition of using eye movements in the investigation of single word processing, far fewer studies have employed this method in the context of strings above word level, such as formulaic sequences. These studies can be divided into two broad categories: (1) the studies looking at figurative language processing and (2) the studies looking at compositional, literal sequences. 


\subsection{Processing of figurative formulaic sequences}

The eye-tracking studies on figurative language such as idioms, have for most part addressed the following questions: Do L1 and proficient L2 speakers process idioms faster than control phrases? How is an idiom's dominant figurative meaning processed relative to the literal one (in ambiguous idioms) in $\mathrm{L} 1$ and $\mathrm{L} 2$ ? And, finally, what is the role of $\mathrm{L}_{1}$ in $\mathrm{L}_{2}$ idiom processing?

In the earliest such study, Underwood, Schmitt and Galpin (2004) investigated idiom processing in L1 and L2 in a story context. They selected a pool of idioms that were between four and eight words in length (honesty is the best policy). The target idioms were matched with novel phrases (it seems that his policy of ...). Underwood et al. (2004) found that the shared words in the idiom condition were read faster than in control phrases in L1, as suggested by fewer and shorter fixations. The authors took their results to suggest that L1 participants were better able to predict the target word within the idiom compared to the control phrase. Interestingly, a similar reading pattern was observed for L2 participants in the number of fixations measure, but not in the fixation duration measure. Underwood et al. (2004) proposed that when an idiom is read, its constituents become predictable as the reader moves towards the second half of the phrase, with the final word being almost redundant. That is, an idiom is recognised as a familiar phrase before the reader has reached the end, which is why the idiom-final word is read faster than the same word in a control phrase. In addition, Underwood et al. (2004) reported that some terminal words in the idiom condition were skipped altogether.

Following up on the work by Underwood et al., (2004), Siyanova-Chanturia, Conklin, and Schmitt (2011) investigated ambiguous idiom comprehension in L1 and L2. Three conditions were looked at: figurative interpretation of an ambiguous idiom (at the end of the day - 'eventually'), literal interpretation of an ambiguous idiom (at the end of the day - 'in the evening'), and a novel phrase (at the end of the war). Because the focus was on frequent and predictable phrases, in a norming procedure the authors obtained a recognition point of the target idioms (a point at which an idiom is recognised as a conventional expression). Thus, the data specific to three regions were analysed: the entire idiom, before and after the recognition point. The study used three eye-tracking measures: first pass reading time (early measure), total reading time (late measure), and fixation count (not a measure of processing time). In addition, the target items' immediate context was matched as closely as possible across the conditions.

It was found that L1 speakers read idioms faster than control phrases (at the end of the day vs. at the end of the war), as suggested by shorter fixation duration and fewer fixations on the former. Further, no differences were found in L1 
reading of the figurative versus literal meaning. A different pattern of results was observed for L2 readers. First, no differences were found in the reading times for idioms versus novel phrases. Second, the processing of L2 idioms used figuratively was more taxing, as suggested by longer fixation duration and more fixations on figurative uses versus literal ones. This processing cost associated with non-literal language appeared greater before the recognition point. Notably, Siyanova-Chanturia et al. (2011a) observed a different pattern of results for early versus late measures. Where significant differences were found in the late measure (total reading time), they were not observed in the early measure (first pass reading time). The authors took this finding to suggest that early measures might not be as sensitive to the mechanisms involved in multi-word information processing as late measures.

\subsubsection{L1 influence on L2 idiom processing}

More recently, eye-tracking research has focused on an important but still relatively understudied issue of $\mathrm{L}_{1}$ influence on $\mathrm{L} 2$ idiom processing. While $\mathrm{L}_{1}$ influence on the processing of formulaic language in a L2 has been researched in some detail (Wolter \& Gyllstad, 2011; Wolter \& Yamashita, 2015), eye movements have only recently begun to be employed in this domain. Carrol and Conklin (2015) investigated idiom priming effects by looking at the processing of translated Chinese idioms by native speakers of Chinese in L2 English. In Experiment 1, these researchers compared the reading of English idioms in L1 and L2 (Chinese) versus controls, and the reading of translated Chinese idioms presented in L2 English versus controls. As expected, English speakers showed facilitation for the idiom-final word compared to a control word. Interestingly, this effect was most apparent in the likelihood of final-word skipping in idioms (31\%). In addition, the advantage for idioms was evident in late, rather than early measures, in line with Siyanova-Chanturia et al. (2011a). Unlike English speakers, Chinese speakers showed no effect for L2 English idioms, consistent with earlier research. Importantly, in the analysis of translated Chinese idioms and their controls, the authors observed a clear difference across a range of measures in Chinese (but not English) speakers, suggesting that cross-linguistic influence played a role in a more facilitative processing of known L1 idioms (even though they were presented in L2).

In Experiment 2, using a comparable pool of participants, Carrol and Conklin (2015) focused on the processing of the literal versus figurative meaning in ambiguous English and Chinese idioms. In line with previous research, English speakers read figurative and literal senses of English idioms equally fast, showing no significant differences in any of the early or late measures examined (i.e. first fixation duration, total reading time, fixation counts, and regression duration). 
Interestingly, Chinese speakers showed a comparable pattern in a L1 and L2: the literal senses were read more quickly than their figurative counterparts, as indicated by shorter fixation duration and fewer number of fixations, consistent with earlier eye-tracking (Siyanova-Chanturia et al., 2011a) and reaction times evidence (Cieslicka, 2006). Based on the results of the two experiments, the authors concluded that when presented in sentence contexts, L1 idioms "retain some level of cohesion" (p.17) when translated into L2, as suggested by the priming effects observed in Experiment 1. However, at the same time, this did not extend to the figurative meaning of the idiom (Experiment 2), pointing to disassociation between form and meaning.

Finally, Carrol, Conklin and Gyllstad (2016) examined how L1 (Swedish) idiom knowledge was used in L2 (English) idiom comprehension. In this study, highly proficient late L2 learners of English were presented with three kinds of idioms, and their respective controls, embedded in short sentence contexts: English-only idiom (utterances that exist only in English, pick your brains vs. control use your brains), Swedish-only idioms translated into English (utterances that exist only in Swedish, sit inside vs. control stay inside), and congruent idioms with comparable L1/L2 form and meaning (meet your match vs. control win your match). Across various eye-tracking measures, Carrol et al. (2016) observed a clear processing advantage not only for English and congruent idioms, but also for translated L1 idioms, over control phrases. This advantage manifested itself in shorter total reading time and fewer fixations on idiomatic phrases, as well as a greater likelihood of the idiom-final word being skipped. In other words, participants treated L1 (Swedish) idioms presented in a L2 (English) as familiar expressions, despite the fact that idioms in this form do not exist in the L2. The authors also analysed the likelihood of skipping of the idiom-final word. Interestingly, L1 Swedish and L1/L2 congruent idioms, but not L2 English idioms, showed an advantage over literal controls. This may suggest that L2 figurative expressions were not "as well entrenched in the mental lexicon" (p. 433) as the other two kinds. Overall, it was concluded that during L2 idiom comprehension, L1 knowledge is automatically activated from the earliest stages of processing.

\subsection{Processing of compositional formulaic sequences}

The eye-tracking studies that have investigated the processing of compositional and literal formulaic sequences have sought to answer the following questions: What is the role of phrasal frequency in $\mathrm{L}_{1}$ and $\mathrm{L}_{2}$ processing? What is the role of adjacency: Do non-adjacent formulaic sequences exhibit a processing advantage compared to novel phrases, just as adjacent items do? 
In one of the earliest such studies, Siyanova-Chanturia, Conklin and van Heuven (2011) investigated readers' sensitivity to phrasal frequency during online language processing. To this aim, these authors looked at the comprehension of binomial expressions (bride and groom) versus their less frequent reversed forms (groom and bride) in $\mathrm{L}_{1}$ and $\mathrm{L}_{2}$ speakers. The use of binomials allowed the researchers to control for lexical (single word) frequency, length, as well as the syntactic structure. A range of predictors were looked at, including phrasal frequency, phrase type (binomial vs. reversed), and proficiency. Proficiency and phrase type were found to interact in the three measures (same measures were analysed as in Siyanova-Chanturia et al., 2011a), showing that proficiency (L1, higher and lower proficiency L2) played an important role in phrasal processing. In addition, phrasal frequency affected reading across the eye-tracking measures. Interestingly, unlike Siyanova-Chanturia, et al. (2011a) where the effect was found in late but not early measures, in Siyanova-Chanturia et al., (2011b), the critical effects were observed across all measures. In this regard, Siyanova-Chanturia (2013), hypothesized that while early measures, such as first pass reading time, might be sensitive to frequency manipulations in shorter sequences (three-word binomial expressions), they may not be sensitive enough when used with longer formulaic sequences, such as idioms (leave a bad taste in your mouth).

Finally, another direction that formulaic language research has recently taken is towards examining the processing of non-adjacent sequences. As Vilkaite (2016) notes, in natural language, non-adjacent sequences are as frequent, if not more frequent, than adjacent ones. Despite this, few studies to date have explored the issue of adjacency (but see Molinaro, Canal, Vespignani, Pesciarelli \& Cacciari, 2013, for evidence from word-by-word reading). Vilkaite (2016) sought to answer the question of whether or not non-adjacent collocations were processed faster than controls, and to compare the magnitude of the processing advantage, if any, to that of adjacent counterparts. To this aim, four conditions were looked at in sentence contexts: (1) adjacent collocation (provide information), (2) adjacent control (compare information), (3) non-adjacent collocation (provide some of the information), (4) non-adjacent control (compare some of the information). Four measures were analysed: first fixation duration and first pass reading time (early), total reading time, and total fixation count (late). The analysis of the phrase-final word suggested an overall stronger facilitative effect for adjacent items compared to non-adjacent ones. However, this effect varied across the eye-tracking measures. For example, no facilitation was found for non-adjacent collocation in the early measures, while total reading time and fixation count suggested faster processing for non-adjacent collocations than their controls. The author suggested that for the earliest stages of word access and retrieval, collocation status (i.e., frequent vs. novel phrase) did not affect processing, while it did in the later stages 
of processing. The analysis of the whole phrase showed consistently faster reading times for collocations than their controls, irrespective of adjacency.

In a follow-up study, Vilkaite and Schmitt (2017) employed the same paradigm and stimuli to investigate the processing of adjacent and non-adjacent collocations in L2 speakers. Akin to L1 readers in Vilkaite (2016), L2 readers read adjacent collocations faster than controls. However, unlike L1 readers in Vilkaite (2016), L2 readers in the follow-up study showed almost no processing advantage for non-adjacent items relative to their controls. Taken together, these findings suggest that adjacency differently affects collocation processing in more and less proficient language users.

As the above review suggests, only a handful of studies have employed eye movements to investigate the processing of strings longer than a single word. The sequences investigated in the studies reviewed above vary in figurativeness and literality, compositionality, frequency and length, congruency with L1 and adjacency. In that, they are heterogeneous. Together, however, these studies point to important processing advantages for formulaic sequences (of various kinds) over novel phrases in L1 and, to a lesser degree, in L2, as well as highlight an important role of L1 during $\mathrm{L}_{2}$ formulaic processing. It is also noteworthy that the use of eye movements has been key in teasing apart early versus late stages of processing, which is particularly important for longer stretches of language. For example, Siyanova-Chanturia, et al. (2011b) observed that during the first pass reading time (early measure), figurative and literal idiom interpretations, and their control phrases, were read in a comparable way. However, significant differences between the conditions emerged in late measures, such as total reading time. These authors hypothesized that when the items of interest are longer sequences, such as idioms, early measures may not be sensitive to potential differences between the conditions. Thus, when looking at idioms and other expressions spanning a few words, it is important to look at a variety of early and late eye-tracking measures. This methodology has further allowed researchers to employ more naturalistic reading tasks, such as presenting target stimuli in sentential and story contexts. Providing sufficient context is critical, for example, in the studies where ambiguous idioms are investigated, so as to allow for either the figurative or literal idiom interpretation. Finally, the methodology offers the possibility to examine skippings (words that did not receive fixations) and to split a region of interest into smaller portions (e.g., before and after the recognition point), which further permits a more finegrained analysis of the data. Clearly, eye movements offer unparalleled benefits in the study of on-line processing of formulaic language. 


\section{Learning of single words}

In recognition of the benefits of the eye-tracking technique, vocabulary researchers have recently started to use this method to study the learning of new lexical items from context.

The first of such studies was conducted by Chaffin, Morris, and Seely (2001). In the first experiment, L1 speakers read a sentence containing a pseudoword, a high- or a low-frequency word. The target word was presented in an informative context and followed by a second sentence in which the target word was referred to by a related word (John picked up the guitar/zither/asdor and begun to strum a tune. He played the instrument to relax). Results showed more re-reading, longer total reading time and more regressions on novel words. Importantly, readers spent longer total reading time in the context following the novel words than following the low- or high-familiar words. They found no effect on the related word in the second sentence, which they interpreted as indicating that readers had already inferred the meaning of the novel form in the first sentence. The second experiment showed that when the context of the first sentence was not informative, readers used the information in the second sentence to infer the meaning of the novel word, as indicated by longer fixation duration and higher number of regressions from the related word.

The initial processing of novel lexical items seems to be influenced by word length. Lowell and Morris (2014) found a similar effect of word length on the initial processing of novel words and known words, but a larger length effect on novel words in the multiple-fixation measures. Readers spent more first-pass time re-fixating long novel targets than short ones. These early studies were the first ones to examine the learning of novel lexical items from context. However, they did not use a post-reading vocabulary measure to ascertain whether readers had correctly inferred the meaning of those lexical items.

This gap was addressed by William and Morris (2004), who examined L1 speakers' eye movements while reading sentences containing target words of different levels of familiarity and then assessed their learning of novel items by a post-reading vocabulary test. Similar to Chaffin et al., (2001), results showed that readers spent more initial processing time on novel words than on familiar words. Scores of the vocabulary recognition test showed that there was a systematic relationship between online processing and memory for novel words. Higher accuracy in the recognition test was related to shorter first pass reading time and to longer second pass reading times, indicating a different effect of early and late measures. This negative relationship between second pass reading times and vocabulary scores could be explained by other factors not controlled for in the experimental design (e.g. word length, see Godfroid, Boers, \& Housen, 2013). 
In a recent study, Joseph, Wonnacott, Forber, and Nation (2014) examined the effect of order of acquisition on the incidental learning of new vocabulary from L1 reading. The skilled adult readers in this study read a set of pseudowords embedded 15 times in meaningful sentence contexts over the course of five days. Half of the pseudowords were presented on day 1 of the exposure phase, whereas the other half were presented on day 2. Immediately after the exposure phase, participants read another set of neutral sentences containing the pseudowords, and then completed a vocabulary task in which they were asked to match the pseudowords with their definitions (test phase). Results of the eye-movement data analysis showed that fixation duration (i.e. first fixation duration, single fixation duration, first pass reading time, and total fixation duration) decreased significantly as an effect of exposure, and that order of acquisition had a significant effect on total fixation duration in the test phase, with longer reading times for late-presented words. Early-presented words were also learned more easily, as shown by the tests in the vocabulary task.

Several similar studies have been conducted in the L2 context. In a study by Godfroid et al. (2013), L2 learners read sentences containing a known control word (boundaries), a matched pseudoword (paniplines), or pseudoword followed or preceded by the known word. Learners' acquisition of pseudowords was assessed by an unannounced multiple-choice gap-filling test. Results showed that learners spent more time reading unknown pseudowords than matched control words, as indicated by significantly longer first fixation duration, first pass reading time, second pass reading times, and total fixation duration. Longer total fixations duration on the pseudowords were related to higher recognition scores, showing that total reading time was a significant predictor of the probability of post-test recognition.

More recently, Pellicer-Sánchez (2016) examined the effect of frequency of exposure on the reading and learning of unknown words. L1 and L2 speakers read a story containing six pseudowords repeated eight times throughout the story. Their knowledge of the pseudowords was assessed by means of several post-reading tests (form recall, meaning recall, and meaning recognition). Results showed a decrease in reading times as an effect of frequency of exposure in three of the four measures examined (i.e. first pass reading time, number of fixations and total reading time), in line with the findings of Joseph et al. (2014). After three to four encounters, L2 participants experienced a significant decrease in number and duration of fixations (i.e. first pass reading time, number of fixations and total reading time) on pseudowords, and by the eighth encounter the novel words were read in a similar way to matched known words, as suggested by the lack of difference between the reading of pseudowords and known items in all eye-movement measures examined. The reading behaviour of $\mathrm{L}_{1}$ and $\mathrm{L}_{2}$ participants was 
very similar, with the significant effect of frequency appearing earlier for L1 participants. Results also showed that aggregated reading times (i.e. the sum of fixation duration of all encounters of the same target item) were related to participants' ability to recall the meaning of the pseudowords.

The significant effect of frequency of exposure on the reading of unknown items has more recently been confirmed by Mohamed (2017). The L2 learners in this study read a graded reader which contained 20 pseudowords that varied in the number of repetitions (1-30 exposures). Learners' knowledge of the pseudowords was examined in three post-reading vocabulary tasks (form recognition, meaning recognition, and meaning recall). The study confirmed previous findings that more time is generally spent reading pseudowords and that reading times decrease with subsequent exposures. Aggregated reading times were also a predictor of vocabulary gains. The role of context was further examined and results showed a significant effect of context predictability, with higher context predictability leading to shorter reading times (Mohamed, 2015).

A more fine-grained picture of the process was recently provided by Elgort, Brysbaert, Stevens, and Van Assche (2017). The participants in this study were asked to read a continuous expository text while their eye movements were recorded. The change in reading times on unknown low-frequency target words and high-frequency control words across multiple exposures was compared. Confirming the findings of Pellicer-Sánchez (2016) and Mohamed (2017), results showed that the differences in the reading of the target and control items reduced significantly by the eighth exposure, as indicated by a significant interaction between word type and exposure across all measures examined, i.e. measures of lexical access (first fixation duration, first pass reading time) and measures of word-to-text integration (go-past time, total reading time, total number of fixations and regressions). They expanded previous research by exploring the quality of the lexical representation established from reading. Specifically, they examined whether the meaning representations created could be abstracted from the specific context of the text used in the study and accessed in semantically neutral contexts. After the main reading task, participants were asked to read a set of semantically-neutral sentences that contained the target items while their eye movements were recorded. The processing of target items in these sentences was compared to that of the targets in the last occurrence of the continuous text. Results showed longer reading times and higher number of fixations and regressions in the sentences than in the continuous text, suggesting that the lexical representations of the target words were still weak and partially contextually-bound. No reliable relationship between eye-movement measures on the sentence-reading post-test and meaning recall was observed. 
The studies reviewed above examined the learning of words from written input. Other studies have used eye-tracking to examine the learning of vocabulary from multimodal input. Bisson, van Heuven, Conklin, and Tunney (2015) investigated the effect of visual information on the learning of vocabulary. Participants were presented with foreign language (FL) words one by one in two modes: auditory word with L1 written translation or auditory word with L1 translation and picture. Results showed that pictures led to higher scores in a one-week delayed recall test and that processing time on pictures predicted both recognition and recall scores, showing a relationship between processing time and learning gains.

A different type of multimodal material was examined by Montero Perez, Peters, and Desmet (2015). The learners in this study watched two subtitled videos in two types of captioning (i.e. full captioning and keyword captioning) and under two test announcement conditions (i.e. the incidental condition with unannounced post-tests, and the intentional condition with announced post-tests). Participants' knowledge of the target words was assessed in a series of vocabulary tests (form recognition, clip association, meaning recall, meaning recognition). Eye-movement data showed that test announcement had an effect on processing times on target words, with the intentional group exhibiting longer second pass reading times and total fixation duration than the incidental group. A positive relationship between total fixation duration and form recognition scores was found for the full captioning, intentional group, supporting previous findings that longer reading times seem to be related to higher vocabulary gains. Interestingly, a negative relationship was found between the second pass reading time and form recognition, which is interpreted as reflecting difficulties in the reanalysis of target words.

Studies investigating vocabulary learning have shown the benefits of using eye-tracking to explore the link between online processing and vocabulary gains, as well as the effects of such factors as contextual predictability, contextual informativeness, input enhancement and frequency of exposure. In general, the results of these studies have shown that when L1 and L2 speakers first encounter unknown lexical items in context, they spend a longer time processing them than known items, which could reflect attempts to guess their meaning from context. This initial, more effortful processing decreases as a function of frequency of exposure. Importantly, these studies have shown that there seems to be a positive relationship between reading times and vocabulary gains, with longer reading times related to higher vocabulary gains, providing further direct evidence for the role of increased attention and engagement in learning. 


\section{Learning of formulaic language}

The most recent development in vocabulary research using eye-tracking regards the learning of formulaic sequences. With a focus on the effect of textual enhancement, Choi (2017) examined the processing and learning of collocations. Participants read a passage containing 14 target lexical (big meal) and grammatical (on week-ends) collocations. One group of participants read the experimental text in an enhanced condition (i.e. target collocations boldfaced), while another group read the same text with unenhanced collocations. A recall test (cloze task) was used to measure participants' knowledge of the target collocations before and after the treatment. Results showed that both conditions led to significant learning of target collocations, with the enhanced condition outperforming the unenhanced condition. Eye-movement data showed longer total fixation time and more fixations in the enhanced condition and on the unknown target collocations.

Choi's study was the first one to use eye-tracking to investigate the learning of formulaic sequences from reading. However, the relation between the eye-movement measures (i.e. number of fixations and total fixation duration) and processing on collocations and learning gains was not explored in the study. PellicerSánchez and Siyanova-Chanturia (2016) addressed this gap by investigating the processing and learning of adjective+noun combinations across several encounters in a story context. A group of L1 and L2 speakers of English read a story with six collocations embedded in them. Participants were assigned to one of the following conditions: (1) eight repetitions of adjective-pseudoword collocations (magic salp 'magic ring'), (2) four repetitions of adjective-pseudoword collocations, or (3) eight repetitions of control collocations (magic ring). After the reading, participants completed a battery of tests which assessed their knowledge of the target pseudowords and their collocates (recall and recognition). Analysis of three online measures (i.e. first pass reading time, total reading time, number of fixations) showed longer reading times on novel collocations on the first encounter and a significant decrease in reading times as an effect of exposure, indicating a learning effect of collocations which was distinct from the effect of repetition. In addition, contrary to the patterns found for single word learning, no significant correlations between the eye-movement measures and any of the vocabulary tests were found.

The results of these studies point to interesting similarities between the processing of formulaic sequences and single words. Similar to single word studies, participants spend more time reading unknown collocations the first time they are encountered, and a significant decrease is observed as a result of exposure. Factors such as textual enhancement also seem to lead to more processing of target items. Importantly, the study by Pellicer-Sánchez and Siyanova-Chanturia 
(2016)showed no relationship between reading times and the learning of collocations, suggesting a differential effect between the learning of single words and multiword sequences.

\section{Conclusions and directions for future research}

The eye-tracking research currently available has undoubtedly broadened our understanding of the processing and learning of vocabulary. However, important unresolved issues call for more eye-tracking based vocabulary studies. From this review, a number of gaps and directions for future research can be identified.

\subsection{Processing of words and formulaic sequences}

In terms of the processing of single words, eye-tracking studies examining nonselective access in bilingual readers (i.e. the activation of lexical representations in the L1 when processing words in the L2) have for most part looked at such effects during L2 reading (Whitford et al., 2016). More studies are, however, needed that investigate non-selective access in bilingual readers during L1 reading. In addition, eye-tracking studies investigating cross-linguistic activation have by and large focused on cognate and interlingual homograph processing (Whitoford et al., 2016). Far fewer studies have looked at other factors, such as cross-language orthographic neighbourhood. Overall, because the word has traditionally been the main focus in applied and psycholinguistic research, a rich body of research has accumulated examining the many and varied factors involved in lexical processing, both in L1 and L2.

On the contrary, only a handful of eye-tracking studies have looked at the processing of strings longer than a single word. The review of the processing studies with formulaic sequences suggests that idioms have to date received most attention. Clearly, more studies are needed with a wider range of formulaic sequences; in particular, a greater focus is required on literal and compositional items. Further, it seems that the current research has for most part looked at English formulaic sequences. How formulaic language is processed in other languages (e.g., non-alphabetical languages) is still poorly understood, with little eye-tracking evidence currently available. Another noteworthy point is that the studies reviewed above have by and large looked at advanced learners. How learners of other proficiency levels (e.g. beginner and intermediate) process formulaic language has so far been largely disregarded (but see Hernandez, Costa \& Arnon, 2016 for the evidence from reaction times with intermediate learners). Eye movements can help us shed light not only on the processing advantage for more versus less frequent 
items in early versus late stages of processing, but also investigate any possible interactions with the proficiency factor. Finally, almost all studies reviewed above have employed adjacent sequences as target items (e.g., idioms, binomials, etc.). Yet, as noted by Vilkaite (2016), non-adjacent sequences are as frequent, if not more frequent, than adjacent ones. Thus, the issue of adjacency, both in L1 and L2, will need to be explored more extensively in future studies.

\subsection{Learning of words and formulaic sequences}

As shown in this review, most of the recent eye-tracking studies seem to point at a relationship between reading times and the learning of single words. However, these results should be treated with caution. This connection has only been found in some of the vocabulary post-tests used by the studies reviewed here. There is no agreement as to which measure best captures this relationship. In addition, the eye-movement measure related to vocabulary scores has also varied across the studies (total reading time, aggregated reading time, second pass reading time, etc.). Interestingly, different positive and negative effects have been found for different eye-movement measures. In addition, this relationship has not been found in some of the most recent studies (e.g. Elgort et al., 2017; Pellicer-Sánchez \& Siyanova-Chanturia, 2016). Therefore, we believe this relationship is presently tentative at best and more research is needed before we can make stronger claims about the link between processing times and vocabulary learning.

Further, there is evidence suggesting that methods such as textual enhancement can increase attention to target vocabulary, which seems to support vocabulary learning. Eye-tracking should therefore be used in the future to explore the effectiveness of different instructional methods to increase attention to lexical items.

Eye-tracking has also proved to be useful in examining the role of frequency of exposure in the vocabulary learning process. Future studies should be conducted to further explore the effect of frequency and its interaction with other factors such as the spacing of repetitions, the saliency of target items, or contextual richness.

Importantly, with very few eye-tracking studies looking at learning of vocabulary from different modes of exposure, little is known about how this behaviour changes with learners of different levels of proficiency and of different backgrounds. In addition, the learning studies reviewed here focused on the acquisition of nouns or "pseudonouns". Future studies should look at the learning of other parts of speech. The two studies looking at the learning of formulaic sequences centred on one specific type of compositional formulaic sequence collocations. Further studies should be conducted with other types of formulaic 
sequences (e.g. verb+noun, verb+adv, etc.), as well as figurative expressions, which are likely to add an additional level of difficulty for learners, reflected in different reading patterns and a greater use of contextual cues.

Clearly, more research is needed to build a more complete picture of how the different types of lexical items are processed in L1 and L2 contexts, to clarify the relationship between reading behaviour and possible learning gains, as well as to investigate the effect of a spectrum of learner factors (e.g. reader proficiency, role of L1, etc.) on this process. This clearer understanding of how words and sequences of different features are processed and learned in different contexts and by learners of different characteristics has important pedagogical implications. It informs teaching practices by helping in the selection of the amount and type of materials that are most effective for tangible vocabulary learning to occur. In addition, if the link between reading times and learning outcomes holds true, this would point towards the advantage of modifying teaching materials and using various attention-grabbing and textual enhancement techniques in vocabulary teaching. As this review has endeavoured to demonstrate, eye-tracking provides the necessary means to forge this fuller and finer understanding of vocabulary processing and learning.

\section{References}

Balota, D., Pollatsek, A., \& Rayner, K. (1985). The interaction of contextual constraints and parafoveal visual information in reading. Cognitive Psychology, 17, 364-39o. https://doi.org/10.1016/0010-0285(85)90013-1

Bisson, M.-J., Van Heuven, W., Conklin, K., \& Tunney, R. (2015). The role of verbal and pictorial information in multi-modal incidental acquisition of foreign language vocabulary. Quarterly Journal of Experimental Psychology, 68(7), 1306-1326. https://doi.org/10.1080/17470218.2014.979211

Carpenter, P., \& Just, M. (1983). What your eyes do while your mind is reading. In K. Rayner (Ed.), Eye movements in reading: Perceptual and language processes (pp. 275-307). New York, NY: Academic Press.

Carreiras, M., Perea, M., \& Grainger, J. (1997). Effects of orthographic neighborhood in visual word recognition: Cross-task comparisons. Journal of Experimental Psychology: Learning, Memory, and Cognition, 23, 857-871.

Carrol, G., \& Conklin, K. (2015). Cross language priming extends to formulaic units: Evidence from eye tracking suggests that this idea "has legs". Bilingualism: Language and Cognition, 20(2), 299-317.

Carrol, G., Conklin, K., \& Gyllstad, H. (2016). Found in translation: The influence of L1 on the processing of idioms in L2. Studies in Second Language Acquisition, 38(3), 403-443.

Chaffin, R., Morris, R. K., \& Seely, R.E. (2001). Learning new word meanings from context: A study of eye movements. Journal of Experimental Psychology: Learning, Memory, and Cognition, 27, 225-235. 
Choi, S. (2017). Processing and learning of enhanced English collocations: An eye-movement study. Language Teaching Research, 21(3), 403-426.

Cieslicka, A. (2006). Literal salience in on-line processing of idiomatic expressions by second language learners. Second Language Research, 22, 115-144. https://doi.org/10.1191/0267658306sr2630a

Coltheart, M., Davelaar, E., Jonasson, J. T., \& Besner, D. (1977). Access to the internal lexicon. In S. Dornic (Ed.), Attention and performance VI (pp. 535-555). Hillsdale, NJ: Lawrence Erlbaum Associates.

Conklin, K., \& Pellicer-Sánchez, A. (2016). Using eye-tracking in applied linguistics and second language research. Second Language Research, 32(3), 453-467.

Cop, U., Dirix, N., Drieghe, D., \& Duyck, W. (2017). Presenting GECO: An eye-tracking corpus of monolingual and bilingual sentence reading. Behavior Research Methods, 49(2), $602-615$.

Desmet, T., \& Duyck, W. (2007). Bilingual language processing. Language and Linguistics Compass, 1(3), 168-194. https://doi.org/10.1111/j.1749-818X.2007.00008.x

Dirix, N., Cop, U., Drieghe, D., Duyck, W., \& Hartsuiker, R. J. (2017). Cross-lingual neighborhood effects in generalized lexical decision and natural reading. Journal of Experimental Psychology: Learning, Memory and Cognition, 43(6), 887-915.

Duyck, W., Van Assche, E., Drighe, D., \& Hartsuiker, R. (2007). Visual word recognition by bilinguals in a sentence context: Evidence for nonselective lexical access. Journal of Experimental Psychology: Learning, Memory, and Cognition, 33(4), 663-679.

Elgort, I., Brysbaert, M., Stevens, M., \& Van Assche, E. (2017). Contextual word learning during reading in a second language: An eye movement study. Studies in Second Language Acquisition, 1-26. https://doi.org/10.1017/So272263117000109

Godfroid, A., Boers, F., \& Housen, A. (2013). An eye for words: Gauging the role of attention in incidental L2 vocabulary acquisition by means of eye tracking. Studies in Second Language Acquisition, 35, 483-517. https://doi.org/10.1017/So272263113000119

Grainger, J., O'regan, J.K., Jacobs, A.M., \& Segui, J. (1989). On the role of competing word units in visual word recognition: The neighborhood frequency effect. Perception \& Psychophysics, 45(3), 189-195. https://doi.org/10.3758/BF03210696

Inhoff, A. W., \& Rayner, K. (1986). Parafoveal word processing during eye fixations in reading: Effects of word frequency. Perception and Psychophysics, 40, 431-439. https://doi.org/10.3758/BF03208203

Joseph, H. S. S. L., Wonnacott, E., Forber, P., \& Nation, K. (2014). Becoming a written word: Eye movements reveal order of acquisition effects following incidental exposure to new words during silent reading. Cognition, 133, 238-248. https://doi.org/10.1016/j.cognition.2014.06.015

Kliegl, R., Grabner, E., Rolfs, M., \& Engbert, R. (2004). Length, frequency, and predictability effects of words on eye movements in reading. European Journal of Cognitive Psychology, 16, 262-284. https://doi.org/10.1080/09541440340000213

Lemhöfer, K., Dijkstra, T., \& Michel, M. C. (2004). Three languages, one echo: Cognate effects in trilingual word recognition. Language and Cognitive Processes, 19, 585-611. https://doi.org/10.1080/01690960444000007

Libben, M. R., \& Titone, D. A. (2009). Bilingual lexical access in context: Evidence from eye movements during reading. Journal of Experimental Psychology: Learning, Memory, and Cognition, 35, 381-390. 
Lowell, R., \& Morris, R. K. (2014). Word length effects on novel words: Evidence from eye movements. Attention, Perception, and Psychophysics, 76, 179-189. https://doi.org/10.3758/s13414-013-0556-4

McConkie, G., \& Rayner, K. (1975). The span of the effective stimulus during a fixation in reading. Perception and Psychophysics, 17, 578-586. https://doi.org/10.3758/BFo3203972

Mohamed, A.A. (2015). The roles of context and repetition in incidental vocabulary acquisition from $L 2$ reading: An eye movement study (Unpublished doctoral dissertation). Michigan State University.

Mohamed, A. A. (2017). Exposure frequency in L2 reading. An eye-movement perspective of incidental vocabulary learning. Studies in Second Language Acquisition. Online first view, https://doi.org/10.1017/S0272263117000092

Molinaro, N., Canal, P., Vespignani, F., Pesciarelli, F., \& Cacciari, C. (2013). Are complex function words processed as semantically empty strings? A reading time and ERP study of collocational complex Prepositions. Language and Cognitive Processes, 28(6), 762-788. https://doi.org/10.1080/01690965.2012.665465

Montero Perez, M., Peters, E., \& Desmet, P. (2015). Enhancing vocabulary learning through captioned video: An eye-tracking study. The Modern Language Journal, 99(2), 308-328. https://doi.org/10.1111/modl.12215

Pellicer-Sánchez, A. (2016). Incidental L2 vocabulary acquisition from and while reading: An eye tracking study. Studies in Second Language Acquisition, 38, 97-130.

Pellicer-Sánchez, A., \& Siyanova-Chanturia, A. An eye-tracking investigation of incidental collocation learning during reading. Paper presented at the Annual conference of the American Association of Applied Linguistics, 8-12 April 2016, Orlando, Florida.

Perea, M., \& Pollatsek, A. (1998). The effects of neighborhood frequency in reading and lexical decision. Journal of Experimental Psychology: Human Perception and Performance, 24, 767-779.

Pollatsek, A., Perea, M., \& Binder, K. (1999). The effects of "neighborhood size" in reading and lexical decision. Journal of Experimental Psychology: Human Perception and Performance, $25,1142-1158$.

Radach, R., \& Kennedy, A. (2004). Theoretical perspectives on eye movements in reading: Past controversies, current issues, and an agenda for the future. European Journal of Cognitive Psychology, 16, 3-26. https://doi.org/10.1080/09541440340000295

Rayner, K. (2009). The 35th Sir Frederick Bartlett Lecture: Eye movements and attention during reading, scene perception, and visual search. Quarterly Journal of Experimental Psychology, 62, 1457-1506. https://doi.org/10.1080/17470210902816461

Rayner, K., \& Bertera, J.H. (1979). Reading without a fovea. Science, 206, 468-69. https://doi.org/10.1126/science.504987

Rayner, K., \& Duffy, S. (1986). Lexical complexity and fixation times in reading: Effects of word frequency, verb complexity, and lexical ambiguity. Memory and Cognition, 14, 191-201. https://doi.org/10.3758/BFo3197692

Rayner, K., \& Well, A. (1996). Effects of contextual constraint on eye movements in reading: A further examination. Psychonomic Bulletin and Review, 3, 504-509. https://doi.org/10.3758/BFo3214555

Roberts, L., \& Siyanova-Chanturia, A. (2013). Using eye-tracking to investigate topics in L2 acquisition and L2 sentence and discourse processing. Studies in Second Language Acquisition, 35(2), 213-235. https://doi.org/10.1017/S0272263112000861 
Siyanova-Chanturia, A. (2013). Eye-tracking and ERPs in multi-word expression research: A state-of-the-art review of the method and findings. The Mental Lexicon, 8(2), 245-268. https://doi.org/10.1075/ml.8.2.06siy

Siyanova-Chanturia, A., Conklin, K., \& Schmitt, N. (2011a). Adding more fuel to the fire: An eye-tracking study of idiom processing by native and nonnative speakers. Second Language Research, 27, 251-272. https://doi.org/10.1177/0267658310382068

Siyanova-Chanturia, A., Conklin, K., \& van Heuven, W. (2011b). Seeing a phrase 'time and again' matters: The role of phrasal frequency in the processing of multi-word sequences. Journal of Experimental Psychology: Language, Memory and Cognition, 37, 776-84.

Titone, D., Libben, M., Mercier, J., Whitford, V., \& Pivneva, I. (2011). Bilingual lexical access during L1 sentence reading: The effects of L2 knowledge, semantic constraint, and L1-L2intermixing. Journal of Experimental Psychology: Learning, Memory, and Cognition, $37,1412-1431$.

Underwood, G., Schmitt, N., \& Galpin, A. (2004). The eyes have it: An eye-movement study into the processing of formulaic sequences. In N. Schmitt (Ed.), Formulaic sequences (pp.153-72). Amsterdam: John Benjamins. https://doi.org/10.1075/Illt.9.ogund

Van Assche, E., Drieghe, D., Duyck, W., Welvaert, M., \& Hartsuiker, R. J. (2011). The influence of semantic constraints on bilingual word recognition during sentence reading. Journal of Memory and Language, 64, 88-107. https://doi.org/10.1016/j.jml.2010.08.006

Van Assche, E., Duyck, W., Hartsuiker, R. J., \& Diependaele, K. (2009). Does bilingualism change native-language reading? Psychological Science, 20, 923-927. https://doi.org/10.1111/j.1467-9280.2009.02389.x

Van Heuven, W. J. B., Dijkstra, T., \& Grainger, J. (1998). Orthographic neighborhood effects in bilingual word recognition. Journal of Memory and Language, 39, 458-483. https://doi.org/10.1006/jmla.1998.2584

Vilkaite, L. (2016). Are nonadjacent collocations processed faster? Journal of Experimental Psychology: Learning, Memory and Cognition, 42(10), 1632-1642.

Vilkaite, L., \& Schmitt, N. (2017). Reading collocations in an L2: Do collocation processing benefits extend to non-adjacent collocations? Applied Linguistics. https://doi.org/10.1093/applin/amx030

Von Studnitz, R.E., \& Green, D. W. (2002). The cost of switching language in a semantic categorization task. Bilingualism: Language and Cognition, 5, 241-251. https://doi.org/10.1017/S1366728902003036

Whitford, V., \& Titone, D. (2012). Second language experience modulates first- and secondlanguage word frequency effects: Evidence from eye movement measures of natural paragraph reading. Psychonomic Bulletin \& Review, 19, 73-80.

https://doi.org/10.3758/s13423-011-0179-5

Whitford, V., Pivneva, I. \& Titone, D. (2016). Eye movement methods to investigate bilingual reading. In R. R. Heredia, J. Altarriba \& A. B. Cieślicka (Eds.), Methods in bilingual reading comprehension research (pp. 183-211). Dordrecht: Springer.

https://doi.org/10.1007/978-1-4939-2993-1_8

William, R.S., \& Morris, R. K. (2004). Eye movements, word familiarity, and vocabulary acquisition. European Journal of Cognitive Psychology, 16, 312-339. https://doi.org/10.1080/09541440340000196 
Wolter, B., \& Gyllstad, H. (2011). Collocational links in the L2 mental lexicon and the influence of L1 intralexical knowledge. Applied Linguistics, 32, 430-449. https://doi.org/10.1093/applin/amro11

Wolter, B., \& Yamashita, J. (2015). Processing collocations in a second language: a case of first language activation? Applied Psycholinguistics, 36, 1193-1221.

https://doi.org/10.1017/S0142716414000113

\title{
Address for correspondence
}

\author{
Ana Pellicer-Sánchez \\ Department of Culture, Communication \& Media \\ UCL Institute of Education, University College London \\ Room 618, 20 Bedford Way \\ London $\mathrm{WC1} \mathrm{H}$ oAL \\ UK \\ a.pellicer-sanchez@ucl.ac.uk
}

